\title{
Dieu-Père/Dao-Mère : dualismes occidentaux et chinois
}

God the Father and Dao the Mother: Western and Chinese Dualisms

神-父/道-母 : 中西二元論

John Lagerwey

\section{OpenEdition}

\section{Journals}

Édition électronique

URL : http://journals.openedition.org/extremeorient/212

DOI : 10.4000/extremeorient.212

ISSN : 2108-7105

\section{Éditeur}

Presses universitaires de Vincennes

Édition imprimée

Date de publication : 1 janvier 2012

Pagination : 137-158

ISSN : 0754-5010

Référence électronique

John Lagerwey, « Dieu-Père/Dao-Mère : dualismes occidentaux et chinois », Extrême-Orient ExtrêmeOccident [En ligne], Hors-série | 2012, mis en ligne le 01 janvier 2015, consulté le 07 mai 2019. URL : http://journals.openedition.org/extremeorient/212 ; DOI : 10.4000/extremeorient.212 


\title{
Dieu-Père/Dao-Mère : dualismes occidentaux et chinois
}

\author{
John Lagerwey
}

Car la lettre tue, mais l'Esprit vivifie.

II Corinthiens 3, 6 .

Cet essai comportera trois parties, correspondant à trois thèses: 1) le dualisme est à la fois universel et particulier (culturel) ; 2) l'opposition Dieu-Père/DaoMère résume au mieux les différences entre dualismes occidentaux et chinois ; 3) l'Histoire peut se comprendre comme une rationalisation patriarcale aux contours déterminés par le «pli» particulier à une culture donnée.

J'ajoute ceci: le dualisme étant de ces dispositifs intellectuels qui nous «colle[nt] au corps», en parler sans évoquer son propre itinéraire serait mentir.

\section{Dualismes occidentaux et chinois}

Dans la Loi, est-ce la lettre qui importe ou l'esprit? La réponse, en Occident, semble évidente : c'est l'esprit, car l'esprit représente l'intention du législateur. Pourtant, dans une cour de justice, si les intentions du meurtrier sont d'une importance capitale pour distinguer entre un meurtre au premier degré et un homicide involontaire, c'est l'acte de meurtre qui déclenche le processus légal. Et, si Jésus affirme que celui qui commet l'adultère dans son cœur est déjà coupable d'adultère, ce n'était pas le point de vue du système juridique lorsque celui-ci s'occupait encore de pareilles choses.

D'ailleurs, ce n'est pas parce que la lettre tue que saint Paul rejette la Loi: ses épîtres véhiculent un nombre impressionnant de règles et de consignes, car «ni les débauchés, ni les idolâtres, ni les adultères, ni les efféminés, ni les homosexuels, ni les voleurs, ni les cupides, ni les ivrognes, ni les outrageux, ni les ravisseurs n'hériteront le royaume de Dieu» (I Corinthiens 6, 9,10). Aussi pense-t-il que «l'homme ne doit pas se couvrir la tête, puisqu'il est l'image et la gloire de Dieu, tandis que la femme est la gloire de l'homme... C'est pourquoi la femme, à cause des anges, doit avoir sur la tête une marque de l'autorité dont 
elle dépend... Jugez-en vous-mêmes : est-il convenable qu'une femme prie Dieu sans être voilée ?» (I Corinthiens 11, 7, 10, 13) Il ne rejette donc pas du tout l'idée d'obéissance à la Loi, loin de là ${ }^{1}$, mais il y a deux lois, celle de la chair et du péché et celle de Dieu: «L'affection de la chair, c'est la mort, tandis que l'affection de l'Esprit, c'est la vie et la paix ; car l'affection de la chair est inimitié contre Dieu... Si l'Esprit de celui qui a ressuscité Jésus d'entre les morts habite en vous, celui qui a ressuscité Christ d'entre les morts rendra aussi la vie à vos corps mortels par son Esprit qui habite en vous » (Romains 8, 6, 11). Ou encore : «Ainsi en est-il de la résurrection des morts. Le corps est semé corruptible; il ressuscite incorruptible... Il est semé corps naturel, il ressuscite corps spirituel» (I Corinthiens 15, 42, 44). S'il y a donc bien, chez saint Paul, un dualisme de la lettre-chair et de l'esprit-vie, ce n'est pas l'opposition qui nous est familière entre corps et âme ou entre matière et esprit, puisque le but ultime est la résurrection du corps, quand bien même ce corps serait un « corps spirituel».

Il n'empêche qu'en Occident, nous vivons toujours tiraillés entre deux formes d'hérésie, matérialiste ou spiritualiste, y compris par rapport à l'interprétation des passages que nous venons de lire: pour les «fondamentalistes », il faut prendre «à la lettre » l'idée de résurrection du corps; pour les «libéraux», mieux vaut imaginer ce que pourrait être un «corps spirituel» en analysant la rhétorique du texte, afin d'en comprendre le «sens », l' «esprit», l' «intention».

Est-ce ainsi en Chine? La réponse rapide est non: puisqu'il n'y a pas de lettres, comment pourrait-il y avoir un esprit qui s'y oppose?

Je donne ainsi une tournure derridienne à l'interprétation de l'affirmation péremptoire de Paul selon laquelle «la lettre tue mais l'esprit vivifie»: lorsque, dans La Voix et le Phénomène et De la grammatologie, Derrida dénonce le préjugé métaphysique occidental, depuis Platon jusqu'à Lévi-Strauss en passant par Rousseau - contre l'écriture, il critique aussi l'opposition constitutive entre «lettre morte» et «vive voix». Dans d'admirables pages sur Platon, il montre les équivalences, dans le système platonicien, entre les notions de père, de soleil, de voix et de vie, par opposition à la mère, la lune, l'écriture et la mort.

Derrida ne se penche guère sur les origines bibliques du dualisme occidental. C'est dommage, car Augustin, après sa conversion du manichéisme au

1. La lecture antinomienne de Paul fait penser à celle, plus récente, du Chan, où les interprètes ont pris le mythe du Chan pour sa réalité. Ainsi, T. Griffith Foulk, l'un des grands déconstructeurs de ce mythe du Chan, après avoir souligné le fait que «tous les monastères publics sous les Song, y compris les monastères Chan, avaient les mêmes dispositions architecturales pour la pratique de la méditation, l'étude des écritures et la récitation des écritures en groupe», conclut que l'«emphase sur le caractère immédiat de l'expérience de l'éveil et non pas sur les textes est un mythe»; voir Foulk (1999): 287 , note 1 et 220 . 
christianisme, allait faire la synthèse de la théologie paulinienne et de l'ontologie platonicienne. Son dualisme, dans La Cité de Dieu et la cité des hommes, par exemple, reste un dualisme éthique, comme chez Paul, mais il devient aussi, à l'aune de Platon, un dualisme ontologique et, partant, l'Occident latin pensera en termes à la fois éthiques et métaphysiques les couples lexicaux corps/âme, matière/esprit, femme/homme, politique/religion (ou État/Église) en en donnant toujours une valeur négative au premier terme du couple.

En employant les catégories aristotéliciennes d'essence et d'accident, tous deux toujours présents en chaque chose, Thomas d'Aquin a détourné le regard philosophique du Ciel des Idées vers le monde naturel des choses. Conjugué à sa distinction ontologique entre nature et surnature et épistémologique entre raison et révélation, l'aristotélisme de Thomas d'Aquin créa ainsi les conditions de l'avènement de la science moderne car, une fois qu'au XVIII ${ }^{\mathrm{e}}$ siècle les Philosophes avaient fait de Dieu un horloger, le règne de la raison souveraine pouvait commencer et la modernité «laïque » et «athée» voir le jour. Mais cela n'a rien changé au dualisme du corps et de l'esprit, au contraire: l'esprit humain cessait peut-être de s'occuper d'Idées désincarnées pour se pencher sur les essences des choses, mais la démarche d'abstraction - qui consiste à soustraire à la chose tout ce qui ne fait pas partie de son «essence»-restait la même. Pire: les «accidents », c'était en fait tout ce qui avait trait aux «corps» des choses, leur situation dans l'espace, leur couleur, etc., bref tout ce que la perception humaine pouvait «surajouter» aux choses de subjectif, de non intrinsèque. C'est ainsi qu'on finit par distinguer entre «qualités premières »-essentielles - des choses et «qualités secondes», sensorielles, ou entre l'objectivité mathématisable et la subjectivité extrinsèque. Le corps dans le thomisme, sujet aux accidents du temps et de l'espace, est plus que jamais une entrave à l'esprit qui recherche la Raison.

C'est ainsi qu'on peut mettre sur le compte du rationalisme thomiste et l'avènement de la science moderne «matérialiste» et la théologie moderne «fondamentaliste», toutes deux farouchement accrochées à des corps, pour en extraire le sens. La première commença par lire avec confiance le Livre de la Nature comme on lisait autrefois la Parole de Dieu, mais finit par rejeter toute idée de révélation et de surnature pour devenir «matérialiste»; la seconde commença, au temps de Gutenberg et de la Réforme, par lire avec confiance et même joie le Livre de la Parole redevenu accessible, pensant retrouver le pur Logos longtemps obscurci, mais finit, au XIX ${ }^{e}$ siècle, par se trouver acculée, par les progrès de la science herméneutique, au choix évoqué ci-dessus entre une lecture littérale «fondamentaliste»- ou «libérale», «spirituelle» du texte biblique.

Ainsi, les anciens dualismes de la lettre et de l'esprit prenaient-ils de nouveaux atours, se renversaient même puisqu'on finit par se trouver pris dans les rets d'une controverse absurde entre «matérialisme» scientifique et «subjectivité» 
religieuse, puis entre «spiritualisme» hégélien et «matérialisme» marxiste. Mais, à travers tous ces tours et détours de l'histoire, le dualisme occidental restait résolument fidèle à lui-même, ainsi qu'on peut le voir par l'opposition hégélienne entre matérialisme et spiritualisme, où le progrès religieux se définit en termes de degré de «rationalité », c'est-à-dire d'abstraction - jusqu'à la noosphère de Teilhard de Chardin -, de déterritorialisation et de dématérialisation. Dans cette vision, le christianisme (protestant s'entend) était la plus rationnelle/ spirituelle des religions, et cela expliquait pourquoi l'Occident était en passe de devenir le maître du monde, grâce aux progrès scientifiques qui dépendaient de la même Raison: «Femme, lui dit Jésus, crois-moi, l'heure vient où ce ne sera ni sur cette montagne ni à Jérusalem que vous adorerez le Père. Vous (les Samaritains) adorez ce que vous ne connaissez pas; nous (les Juifs) nous adorons ce que nous connaissons, car le salut vient des Juifs. Mais l'heure vient, et elle est déjà venue, où les vrais adorateurs adoreront le Père en esprit et vérité, car ce sont là les adorateurs que le Père demande. Dieu est Esprit, et il faut que ceux qui l'adorent l'adorent en esprit et en vérité» (saint Jean 4, 21-24). Ainsi, la science des religions naissante (à l'École Pratique des Hautes Études [EPHE] notamment) opposait matérialisme rituel hébraïque et religion spirituelle chrétienne, comme plus tard l'anthropologie opposera rite et mythe. Si Lévi-Strauss nous a donné quatre gros volumes de «mythologiques», pourquoi le sacrifice l'a-t-il tant dérangé ? Et pourquoi, après trente ans d'engouement pour les rites, n'avons-nous toujours pas de «ritologiques»?

Pourquoi faut-il commencer par l'Occident pour parler de la Chine ? - pour la simple raison qu'en tant qu'Occidentaux, déroutés par l'étrangeté de la Chine, par son altérité, nous risquons de la juger selon nos propres critères. Que ce soit en bien ou en mal, peu importe: nous lui appliquerons nos catégories habituelles, même quand on y prend garde. Ainsi, François Jullien, héritier de la vision jésuite de la Chine, y trouve un monde où la Raison philosophique peut se déployer sans recours à la Révélation, au «transcendant»: sa pensée est «organique », et met en scène un «procès » du monde, comme dans la physique moderne. D'autres, souvent des ex-maoïstes, sont plutôt perturbés par le fait que l'État chinois se comporte en Église, et organise régulièrement la chasse aux sorcières. Moi-même, dans une incarnation antérieure, j'ai aimé Zhuangzi par-dessus tout car il était «nominaliste». Lorsqu'il disait: «Où puis-je trouver quelqu'un qui a oublié les mots pour avoir un mot avec lui ?», j'y lisais une libération spirituelle encore plus radicale que celle du christianisme, car fondée non pas sur l'expérience spirituelle mais sur la logique et une épistémologie de l'intuition. Il est difficile d'aller en Chine sans tomber amoureux d'une altérité de Narcisse. J'avoue volontiers que j'en suis encore victime. 
Mais on essaie aussi de dépasser ce stade nécessaire du miroir. Dans mon cas, le déclencheur a été la rencontre du taoïsme religieux, dans les cours de Max Kaltenmark et de Kristofer Schipper, à l'EPHE. Tous deux m'apprenaient que «ma Chine» d'Harvard, très jésuite et voltairienne, qui comportait la passion de Zhuangzi et, en une moindre mesure, celle de Laozi, était, comment dire? inexacte: la Chine aussi avait sa propre religion - pas juste le bouddhisme importé puis rejeté (dixit $\mathrm{Hu}$ Shi), mais le taoïsme, et ce taoïsme dit «religieux » n'était pas une version tardive et «superstitieuse » du taoïsme "philosophique» comme on le disait alors, au contraire.

En d'autres termes, déjà épris de taoïsme «philosophique», je me trouvais désormais amoureux du taoïsme honni «religieux». Mais, contrairement au premier, où $\mathrm{j}$ 'avais trouvé un prolongement du spiritualisme de mon éducation familiale calviniste, dans le taoïsme «religieux» je trouvais le contraire. Dans un premier temps, c'étaient des textes dits «alchimiques» qui exerçaient sur moi un étrange attrait: quel pouvait en être la clé, de l'Écrit central du Laozi (Laozi zhong jing), du Classique de la Cour Jaune (Huangting jing), de L'Éveil à la réalité (Wuzhen pian) ou de La Triple Concordance du Livre des mutations (Cantongqi) ? Ces textes ne pouvaient se lire de manière linéaire, mais comment les lire? En les écrivant: en alignant des phrases parallèles, courantes en chinois, mais aussi en alignant les références répétées aux mêmes dieux ou lieux du corps, afin de voir s'il n'y avait pas une logique aux changements qui intervenaient dans les descriptions au fur et à mesure que le texte avançait. C'est ainsi que j'ai trouvé deux logiques: la première, tout simplement celle de la cosmologie chinoise, qui répartit les choses en catégories Yin et Yang, ou les ventilent selon les Cinq Phases (Wuxing); la deuxième, plus difficile à formuler, consistait à modifier le titre d'un esprit du corps selon que cet esprit se trouvait à un endroit ou un autre du corps. Plus tard, j'ai cherché à formaliser la description de ces deux logiques dans «Deux écrits taoïstes». En 1976, lorsque je lisais ces textes pour la première fois, je les comprenais surtout comme des textes «abstraits» et non linéaires (Derrida). Ils représentaient un type de textes qu'aussi bien Mallarmé que Rimbaud - au moment où la musique se faisait «atonale» et que la peinture devenait «abstraite»-avaient cherché mais échoué à produire. Pourquoi seul le langage n'avait-il pas réussi sa désagrégation abstraite, là où les Chinois l'avaient réalisée il y a deux mille ans déjà ? Qu'y avait-il en Occident qui empêchait la création de textes purement formels, sans contenu?

Mais mon engouement pour ces textes n'a pas duré: au printemps de 1977, Schipper invita le maître de Tainan Chen Jung-sheng à l'EPHE. Non seulement assistai-je à ses cours sur le rituel taoïste, mais avec Patrice Fava et deux étudiantes, dans le jardin du 24, rue Hallé, chez Schipper, nous avons fait un rituel avec le maître Chen pour fêter l'anniversaire de Laozi. Puis Chen a fait 
une exposition de ses «talismans» dans une galerie de la rive gauche. Tout le gratin de Paris y était, à inspecter ces grimoires d'Ailleurs et à regarder le maître Chen pratiquer ses rituels sur un autel (tan) aménagé dans la galerie. Je ne me rappelle plus tout ce que j'ai pu penser alors, mais je sais que je me suis posé la question de savoir ce qu'assister à un tel rituel pouvait bien apporter à des Occidentaux qui ne comprenaient ni le rituel ni le chinois? J'allais apprendre par la suite que la question était encore plus pertinente que je ne pensais car, quand j'ai commencé à assister aux rituels du maître Chen à Tainan, à partir de 1980, je me rendis compte que, des cinq taoïstes qui exécutaient ensemble un rituel, seul le maître comprenait ce qu'il faisait: pas très démocratique !

De 1977 à 1980, j'ai surtout travaillé sur les textes Lingbao et Maoshan qui se trouvent dans le Wushang biyao (une encyclopédie taoïste compilée à la demande de l'Empereur vers l'an 574). Pendant ce temps, en cours avec Schipper, nous lisions le Huangshu guodu yi, et je parcourais la thèse de Léon Vandermeersch, «La Voie royale» où, après avoir analysé dans le détail les rituels Shang et avoir réfléchi longuement sur le fait que l'attitude chinoise à l'égard du rituel est radicalement différente de la nôtre, il propose le mot «morphologique» pour résumer la pensée chinoise, par opposition à la pensée occidentale, «téléologique». $\mathrm{Si}$, pour nous, le rite est la forme extérieure («matérielle»), pour les Chinois, c'est la «raison» ou le «principe» (li) qui s'y trouve consigné (un peu comme l'idée de deep structure chez Noam Chomsky). De ce fait, pratiquer un rituel est la seule manière de comprendre une pensée abstraite ou, plutôt, de la faire sienne.

Je continue à penser que l'opposition téléologique/morphologique représente une découverte d'importance majeure pour comprendre la différence entre cultures chinoise et occidentale, et que l'on peut pousser très loin l'analyse de nos différences à partir de ces deux seuls termes. Mais, avant de ce faire, je dois terminer mon récit autobiographique. Pendant que je travaillais sur les textes Lingbao et Maoshan, je lisais beaucoup sur le judaïsme, sur le mysticisme juif (Gershom Scholem), mais également sur sa prière. J'ai appris par cœur les « dixhuit bénédictions » et les récitais quotidiennement pendant plusieurs années. Pratique et lectures m'amenèrent à comprendre qu'il n'y avait absolument pas, dans le judaïsme, de dualisme qui dévalorisait le corporel: ni l'argent ni la sexualité n'y sont considérés avec négativité comme cela a été le cas dans le catholicisme, mais aussi dans le protestantisme (enfin, le protestantisme calviniste a fait sa paix avec l'argent). Même si, d'après Scholem, il y avait toujours un abîme infranchissable, dans le mysticisme juif, entre Créateur et créature - qu'il y avait donc une sorte de dualisme lié à la transcendance absolue du Père -, ce dualisme n'était pas le même que celui de Paul. Surtout, jamais un juif pratiquant n'aurait écrit que «la lettre tue» car la lettre, dans le judaïsme, 
c'est d'abord le texte consonantique sacré, de Dieu. S'il faut, grâce à la tradition orale, vivifier ce texte en lui insufflant les voyelles, comme Dieu insuffla en l'homme la vie, il est néanmoins, ce corps du texte, sacré. D'ailleurs, la prière juive débute ainsi: «L'âme que tu m'as donnée est pure. Tu l'as créée, tu l'as formée, tu l'as insufflée en moi et tu la préserves en moi. Tu me la reprendras un jour pour me la rendre au moment de la Résurrection».

Ainsi, le corps de l'homme est habité par le souffle de vie de Dieu. Finalement, ce n'est peut-être pas si loin de Paul que ça: «Ne savez-vous pas que vous êtes le temple de Dieu, et que l'Esprit de Dieu habite en vous? Si quelqu'un détruit le temple de Dieu, Dieu le détruira; car le temple de Dieu est saint, et c'est ce que vous êtes » (I Corinthiens 3, 16). Donc le corps de Paul-le-chrétien est saint, comme celui de Saul-le-juif. Se pouvait-il que les Gentils, pétris du dualisme de Platon - où l'incorporation est une incarcération empêchant de voir clairement le Ciel des Idées -, avaient mal lu Paul? Je commençais en tout cas à me rendre compte que, en tant qu'Occidental, pour comprendre la Chine, j'avais tout intérêt de partir non pas de la théologie chrétienne ni de la métaphysique de Platon, mais de la prière juive.

Décrié comme orgie par les confucéens et les bouddhistes, le Huangshu guodu yi est un rituel taoïste de mariage dont la chorégraphique complexe permet au couple de construire avec leurs corps un cosmos composé des huit trigrammes. Leur union ultime est le mariage du Yin et du Yang, du Ciel et de la Terre. Or, l'une des expressions les plus prisées du mysticisme juif est le Chant des Chants, dans lequel l'alliance entre Dieu et son peuple est décrite dans des termes charnels qui feraient rougir un chrétien (semble-t-il). Mais si Moïse est, dans le judaïsme, le grand législateur, c'est sa sœur Myriam qui représente le peuple d'Israël dans cette alliance avec Dieu: Israël est la femme de Dieu, c'est pourquoi les prophètes appelaient «prostitution» le fait d'aimer d'autres dieux que Yahweh. Autrement dit, le dualisme juif, ici encore, fait penser au dualisme chinois du Yin et du Yang, du Ciel et de la Terre. C'est-à-dire que c'est un dualisme moniste, ou presque, de couple. D'ailleurs, quand on sait que Marie est grecque pour Myriam, on comprend de suite que, dans la Nouvelle Alliance aussi, il y ait un mariage entre un peuple sur terre et un Père qui est au ciel : l'Église, Nouvel Israël dans la vision du Nouveau Testament, est tout naturellement Myriam, et c'est de cette nouvelle Femme de Dieu que naissent les créatures nouvelles «ensevelies avec lui par le baptême en sa mort, afin que, comme Christ est ressuscité des morts par la gloire du Père, de même nous aussi nous marchions en nouveauté de vie» (Romains 6, 4). 


\section{Dieu-Père/Dao-Mère}

Après cette remise en question du dualisme occidental, qui en situe la véritable source dans la pensée de Platon, ayant au moins suggéré que le dualisme hébraïque est plus proche que le dualisme grec du dualisme chinois, essayons de regarder en quoi les ontologies taoïste et juive sont quand même radicalement différentes. La manière la plus simple de procéder, me semble-t-il, est de faire ce constat: la place de Dieu le Père dans le judaïsme est occupée par Dao la Mère dans le taoïsme ancien. Le Dieu biblique et le Dao ont beaucoup en commun: tous deux sont à la fois présents dans ce qu'il y a de plus infime et englobe tout; tous deux sont la source de tout ce qui existe; tous deux sont «éternels». Mais les différences sont tout aussi éclatantes: là où «prononcer en vain» le nom de Dieu est interdit, le nom du Dao est tout simplement non existant (c'est un qiangming, un nom «forcé»); si le Dieu créateur est séparé de ses créatures par un abîme infranchissable, le Dao est inséparable des choses; tandis que Dieu crée par sa Parole, le Dao se manifeste dans un «enseignement sans paroles» (buyan zhi jiao). En un mot, Dieu est Père; le Dao est Mère. Comment savoir? - le Laozi compare sans cesse le Dao à une mère, à une mère enceinte surtout, ou qui vient d'accoucher. Dans le judaïsme, il faut six jours de la parole de Dieu pour produire le monde; dans le taoïsme, c'est un processus de gestation qui dure neuf mois. Le Zhuangzi, aussi, affirme que la sagesse des anciens était si parfaite qu'ils ne connaissaient pas leur père mais seulement leur mère.

Le Dao, dans le taoïsme religieux, est le corps de Laozi qui, en se transformant, produit l'univers : ses cheveux sont les étoiles, ses yeux le soleil et la lune, et ainsi de suite. Si le Dao est de prime abord l'espace de l'univers, c'est également l'alternance du Yin et du Yang, du wu et du you. En tant que wu (le non-manifeste), c'est le Dao, la Mère; en tant que you (le manifeste), c'est le souffle unique (yuanqi), le Fils. Deux façons différentes de l'imaginer permettent de penser le Dao comme «processus du monde»: soit comme alliance active du couple primordial, où le Vrai Yin est celui qui se trouve dans le Yang et vice versa; soit comme Hundun ou Chaos primordial, où le souffle unique est envisagé sous sa forme dynamique et synthétique. C'est cette dernière qui est contée par le Laozi 42: «Le Dao donne naissance à l'Un, l'Un au Deux, le Deux au Trois et le Trois aux dix mille choses », où l'un est le Ciel et le Yang, le Deux, la Terre et le Yin, et le Trois, soit l'Eau (triade naturelle), soit l'Homme (triade culturelle). C'est en effet - Jullien a raison ici - un processus organique, de synthèse permanente, à la fois ontique et épistémique.

On le voit, Dao-Mère occupe bien la même place fonctionnelle que Dieu-Père dans le système biblique, mais le fait que l'un soit conçu de prime abord comme mère et l'autre comme père conduit à des choix dualistes radicalement opposés : 


\begin{tabular}{|l|l|}
\hline mère & père \\
\hline corps & âme (souffle) \\
\hline matière & esprit \\
\hline espace & temps \\
\hline écriture & voix \\
\hline vue & ouïe \\
\hline rite & mythe \\
\hline choses & mots \\
\hline nature & culture \\
\hline
\end{tabular}

Avant de commenter ce tableau, il faut d'abord en finir avec l'idée que la pensée taoïste (ou chinoise) serait moniste. À mon avis, toute pensée est par définition dualiste puisque, pour exister, elle commence par une distinction entre les mots et les choses. La pensée s'ingénie, ensuite, à fonder le rapport entre ces deux étants. En hébreu, par exemple, le mot dabar veut dire à la fois mot et chose; dans le taoïsme, depuis Laozi, on imagine ces «deux choses »- le non-manifeste et le manifeste - comme ayant «même origine: mystère sur mystère ! La dualité première est, à ce qu'il me semble, celle créée (ainsi Lacan) par l'avènement de la parole, lui-même préparé par l'émergence du «je» comme «moi» dans le miroir, origine de notre désir non déracinable de «redevenir Un». Mais cette dualité se constate aussi dans la différence évidente entre un organisme vivant - un corps, une personne - et un cadavre: au début, Dieu insuffle l'âme; à la fin, on «rend son âme» ou son «dernier soupir» («tu me la reprendras un jour pour me la rendre au moment de la Résurrection»; Luc 23, 46: «entre tes mains je remets mon esprit»).

Le dualisme revêt un caractère universel, et ce qui importe est de voir comment sa définition culturelle organise de manière toujours particulière chaque société et la pensée qui la sous-tend.

Revenons à notre tableau comparatif pour en souligner la logique qui permet de relier, en Chine comme en Occident, les termes en série d'équivalences. Je ne sais si la mère est partout d'abord un corps et le père une parole, mais cela semble bien correspondre au Dao et à Dieu (et au fait que la femme conçoit et porte des enfants, tandis que les hommes ne peuvent concevoir et porter que des idées). Or, un corps prend de la place, comme la parole prend du temps, ce qui produit l'opposition (ou la complémentarité) espace-temps. De même, un rite est d'abord un corps - ou un ensemble de corps - qui s'agite(nt) en un lieu donné, tandis que le mythe est d'abord discours, récit. L'écriture aussi occupe un espace - espace-consonne dans le judaïsme, donc mort s'il reste sans voyelles; «lettre qui tue» pour saint Paul; mais espace de souffles tourbillonnants dans le 
taoïsme, de caractères qui «font signe» ou qui permettent de suivre à la «trace», ou même de faire hurler les démons, selon un mythe ancien de la naissance de l'écriture chinoise. L'écriture occidentale, telle que Derrida la dénonce, est un décalque de la parole; elle est secondaire par rapport à elle. Mais l'écriture chinoise n'est pas une représentation de la parole, et existe autant comme objet esthétique que comme véhicule de discours. C'est pourquoi la calligraphie est devenue le premier art chinois, c'est aussi pourquoi le «talisman» $(f u)$ taoïste - qui représente le nom d'un dieu - est agissant. Un monde où le sens prend d'abord une forme visuelle sous-tend naturellement une pratique religieuse de visualisation, comme celui où le sens est d'abord parole sous-tend la prière: les pratiques symboliques fondamentales, dans l'un et l'autre cas, permettent d'apprivoiser - de faire siennes - les caractéristiques du système de sens. C'est dans cette acceptation très précise que la pratique religieuse est toujours vide de sens en elle-même: son but est de faire corps avec le système culturel, afin de le transcender. Pour le dire autrement, la pratique religieuse opère au niveau du logiciel-système et non pas de l'application.

Pour fonder un système culturel, il va de soi que l'écriture, dès lors qu'elle existe, revêt une importance particulière pour la définition des paramètres du système. Je suggérerais que ce sont justement les caractéristiques des systèmes d'écriture qui expliquent que la pensée chinoise soit devenue morphologique, la pensée occidentale téléologique. Le caractère chinois, on l'a déjà dit, est d'abord forme mystérieuse qui interpelle l'œil, et permet aussi de penser que tout peut être dit par la représentation chorégraphique du rituel. L'écriture alphabétique, par contraste, ne dévoile son sens que lorsqu'elle arrive au terme de son expression: le sens n'est pas immédiat, il part de l'Alpha de l'intention pour atteindre l'Omega du but; il est «buté », et la place qu'occupe la chorégraphie rituelle en Chine est prise, en Occident, par la rhétorique.

Pourquoi le nominalisme a-t-il toujours fait scandale en Occident - au point que l'Église catholique pensait avoir réglé son compte à Calvin lorsqu'elle traitait celui-ci de nominaliste (à cause de son interprétation de la «présence réelle») ? Et pourquoi, chez Zhuangzi, le nominalisme provoque-t-il rires et sentiments de liberté ? Pourquoi, en Occident, le «corps morcelé » est-il (ainsi Lacan) le cauchemar par excellence, tandis que, chez Zhuangzi, il occasionne un rêve de voyage extatique (xiaoyao you)?

Plutôt que de rechercher l'origine de ces différences, je préfère souligner la solidarité de ces attitudes radicalement différentes l'une de l'autre avec les deux systèmes d'écriture: dans un système alphabétique, il n'y a aucun rapport nécessaire entre son et chose (Saussure); autrement dit, le mot, qu'il soit prononcé ou écrit, n'est pas la chose, ne fait que la représenter de manière arbitraire et aléatoire. Dans un tel système, le rapport entre nos représentations mentales 
et les choses pose problème (Platon). Ici, Platon et les Hébreux anciens sont d'accord: il y a un abîme infranchissable, tant que nous sommes ici-bas, dans le corps, entre les Idées (Dieu) et les choses (nous). On n'arrive pas à dire notre mal ; «la vraie vie est absente».

Mais, avec les idéogrammes chinois, où le son est «ajouté», secondaire - il y a des textes taoïstes qui le disent explicitement -, le mot est la chose, et la rend présente. Ceci est particulièrement clair dans la pratique taoïste du $f u$, mais les taoïstes ne font ainsi qu'expliciter la nature profonde du système chinois d'écriture. Ainsi, le problème de la présence réelle, qui a tant divisé les Occidentaux au moment de la Réforme et de la naissance de l'univers Gutenberg (soudain, tout un chacun pouvait s'imaginer interprète, ce qui a toujours été le cas en Chine), n'a jamais dérangé les Chinois. Enfin, si : lorsqu'un disciple demanda à Confucius si les ancêtres étaient réellement présents lorsqu'on leur offre des sacrifices, Confucius répondit: «Faites comme si». Pourquoi? Parce que, si vous faites le rituel de sacrifice dans cet esprit, la question de la présence des esprits ne se pose plus: ils sont là, dans le rite. Il suffit donc de pratiquer le rite dans l'esprit du rite pour que les esprits soient présents, comme il suffit d'écrire un talisman pour que l'esprit ainsi «nommé» soit présent et agisse.

Il y a, ici, stricte équivalence entre la certitude de deux efficacités: celle du talisman et celle du rite. L'un est en fait un acte d'écriture (et non de parole, qui lui est adjointe sous forme d'incantation), l'autre de chorégraphie. Les deux sont des produits de corps agissant dans l'espace: de corps qui (re)produisent des configurations d'énergie. La présence est réelle, il suffit d'être soi-même présent à l'acte. Pas étonnant, dès lors, que Laozi enjoigne de «fermer les portes » et de rentrer au-dedans de soi-même: «Les dix mille choses s'y trouvent». Les choses, ce sont des configurations d'énergie, et toutes ces configurations se trouvent à l'intérieur de mon corps au même titre qu'elles se trouvent en dehors. Mais pourquoi aller les chercher à l'extérieur si elles se trouvent, aussi, à l'intérieur? Pour le dire autrement, l'écriture chinoise représente un univers de corps vivants : de corps dont les principes n'ont pas été extraits pour en faire des abstractions (des Idées) d'un côté et des cadavres de l'autre, mais des organismes dont les principes structurants s'offrent au regard de qui veut bien tourner celui-ci vers l'intérieur de la grotte. C'est un corps «enceint» des dix mille choses: un corps de femme. Ce corps n'a pas besoin de demander à Dieu de «garder son âme», ni de la lui «rendre à la résurrection» (à la fin, buté: téléologique), car c'est un corps rempli de dieux qu'il faut garder, empêcher de partir, voire même conjuguer, comme dans l'alchimie symbolique (neidan).

À cela il faut ajouter une chose: le monde des idéogrammes est un monde d'abord visuel, tandis que le monde de l'alphabet est un monde auditif. Dans un cas, le sens privilégié est la vue, dans l'autre l'ouïe. Or, nous retrouvons ce même 
contraste à l'intérieur du seul Occident, ainsi qu'Erich Auerbach l'a montré dans Mimésis : pour les Grecs, le monde est vision, pour les Hébreux audition. Ainsi, Narcisse est capturé par son image; Edipe s'arrache les yeux. Mais le cœur de la prière juive s'appelle «Schema», «Écoute»: «Écoute, Israël, l'Éternel est notre Dieu, l'Éternel est Un». Ce Dieu éternel se voit, dans le firmament par exemple, mais il est surtout «parole»: «Les cieux racontent la gloire du Seigneur, et le firmament proclame l'œuvre de ses mains. Le jour en parle au jour, et la nuit le fait connaître à la nuit. Point de discours, point de paroles, leur voix ne se fait pas entendre. Leurs accents retentissent par toute la terre, et leurs paroles jusqu'aux extrémités de l'univers » (Psaume 19, 1-5). Qu'est-ce qu'une voix qui «ne se fait pas entendre»? - C'est une voix intérieure: «Et après le feu, un murmure doux et léger. Quand Élie l'entendit, il s'enveloppa le visage de son manteau, il sortit et se tint à l'entrée de la caverne. Et voici, une voix lui fit entendre ces paroles... » (I Rois 19, 12-13).

Donc, de nouveau, dualismes hébraïque et chinois invitent à des comparaisons : chez l'un comme chez l'autre, la présence réelle se trouve à l'intérieur de l'homme; mais chez l'un ce sens s'entend, chez l'autre il se voit (neiguan). Les taoïstes visent à «faire corps avec le Dao» (ti Dao), ou à «conjuguer leur vérité à celle du Dao» (yu Dao hezhen). Tout tourne autour du corps: le corps dans l'espace comme lieu de conjugaison de ce qui est dans le sujet et de ce qui lui correspond de «vrai» dans l'univers, dans le Dao. Dans cette forme du judaïsme qu'on appelle christianisme, «Au commencement était la Parole, et la Parole était avec Dieu, et la Parole était Dieu. Elle était au commencement avec Dieu. Toutes choses ont été faites par elle... Et la Parole a été faite chair, et elle a habité parmi nous, pleine de grâce et de vérité» (saint Jean 1, 1-2, 14). Tout est question de temps, de narration, mais la Parole finit par «prendre chair» et «habiter parmi nous».

Est-ce un pur hasard que ces deux traditions, juive et chinoise, voient dans le cœur le centre de l'être humain? Ou que, dans la tradition philosophique grecque, où le dualisme entre mondes subjectif et objectif, interne et externe, est le plus radical (allégorie de la grotte), l'homme soit défini par son cerveau ? On ne peut mieux résumer la pensée grecque que ne le fait Descartes : «Cogito ergo sum ». Or, ce cogito-là, ce sujet-là, c'est un sujet mâle, un sujet qui n'existe que dans et par ces idées, sans référence au corps, individuel ou social. Le dualisme occidental tant décrié ces derniers temps nous vient des Grecs. Sa recherche d'idées pures, «claires et distinctes », d'esprits purs dans des corps masculins désincarnés, nous a donné la science moderne, son inventivité technologique et la destruction de la planète. 


\section{Le dualisme chinois dans son histoire}

$\mathrm{Si}$, sur le fond, le dualisme chinois accorde davantage de place au féminin, au corps, au rite et à l'espace que le dualisme occidental, l'histoire chinoise obéit à la même règle que l'histoire occidentale: rationalisation (= universalisation, démocratisation et déterritorialisation), intériorisation (= individualisation) et sécularisation, en un mot patriarcalisation. L'histoire appartient au Père.

Pour le dire autrement, la rationalité «laïque» n'est pas la définition de la «modernité» mais de l'histoire tout court. Chaque crise systémique passe par une mise en cause du système de valeurs de la société désormais «archaïque » et conduit à un redéploiement de ces mêmes valeurs sous forme plus rationnelle, intériorisée, universelle, déterritorialisée et «laïcisée». C'est pourquoi on voit souvent les historiens parler d' «humanisme sécularisé» lorsqu'ils évoquent ces moments clés de l'histoire. Pour ne donner que deux exemples en ce qui concerne la Chine, Marc Kalinowski dit ceci des changements qui sont intervenus à l'époque des Royaumes combattants (481 à 220 avant notre ère):

D'autre part, c'est aussi par la bouche des conseillers que s'expriment les attaques ouvertes à l'encontre des arts mantiques. Ce sont eux qui défendent l'idée que le cours des événements, aussi contingent soit-il, repose de manière quasi déterministe sur les tendances bonnes ou mauvaises des hommes et que l'usage excessif des oracles fait obstacle aux prises de décisions plutôt qu'il ne les favorise: «On consulte la tortue pour lever les doutes, pourquoi la consulter alors qu'il n'y a pas lieu de douter !» Aux techniques des scribes et des devins, ils opposent une forme d'intelligence qui consiste à saisir les prémisses de changements futurs dans les situations présentes. Leurs arguments sont empreints d'humanisme confucéen, et l'idéal politique qu'ils défendent demeure au bout du compte celui d'un monarque éclairé qui se soucie du bien public et «ne s'adonne pas inconsidérément à la consultation de la tortue et de l'achillée». ${ }^{2}$

Pour une tout autre époque, celle des Song (960-1279), Yu Ying-shih affirme ceci:

From a historical point of view, the emergence of the Tao-hsïeh community in Sung China may be most fruitfully seen as a result of secularization. There is much evidence suggesting that the Tao-hsieh community was, in important ways, modeled on the Ch'an monastic community. The Neo-Confucian academy, from structure to spirit, bears a remarkable family resemblance to the Ch'an monastery. But the difference is substantial and important. Gradually, quietly, but irreversibly, Chinese society was taking a this-worldly turn with Ch'an masters being replaced by Confucian teachers as spiritual leaders ${ }^{3}$.

2. Kalinowski (2009).

3. Yu (1992): 11. 
Ainsi, dans les deux cas, un ancien système de valeurs qualifié de « religieux » est remplacé par un nouveau système «humaniste» ou «laïque». Est-ce uniquement parce que les historiens contemporains projettent leurs valeurs sur le passé, ou y a-t-il quelque chose de plus profond à l'œuvre? Pour répondre à cette question, regardons les deux cas de plus près.

À l'époque des Royaumes combattants, la royauté Zhou vivait ses derniers jours. Son système de rites, bâti autour du culte des ancêtres royaux, était en pleine déliquescence. Les uns après les autres, les petits États «frères », qui avaient des liens de sang avec la maison royale et qui pouvaient de ce fait participer à la distribution de la viande du sacrifice ${ }^{4}$, se faisaient engloutir par quatre grands États géographiquement périphériques au système, et dont les régimes n'avaient pas ce lien de sang et de sacrifice: Qin, Jin, Qi et Chu. C'est chez eux que s'inventait la future bureaucratie impériale, ainsi que les mythes et rites de nouveaux dieux hors du système des ancêtres Zhou. À la fin, ils ne seront plus que deux, Qin et Chu: le premier l'emportera et fondera le premier empire de l'histoire chinoise, mais cette dynastie des Qin ne survivra pas à son fondateur (221-206 avant notre ère) et sera remplacée par les Han (206 av.-8 apr. ; 25-220), originaires de Chu.

C'est donc pendant cette période des Royaumes combattants que fut menée une réflexion sur les rites - réflexion résumée au mieux par ce dialogue entre Confucius et l'un de ses disciples qu'il nous faut ici citer à nouveau : «Lorsque l'on sacrifie aux ancêtres, sont-ils réellement présents?» demande le disciple. «Faites comme si », répond superbement Confucius. Un autre disciple lui demande: «Pourquoi gaspiller tout cet argent en tuant les boucs?» «- Vous pensez au bouc, moi au rituel. » La même chronique de Zuo (Zuozhuan) que cite Kalinowski pour montrer l'avènement d'un nouveau type de devin - le devin qui fait ses prédictions grâce à sa connaissance de l'histoire et de la nature humaine - comprend aussi toute une série de discours sur le rituel (li) qui nous permettent de comprendre l'attitude de Confucius :

Since time immemorial, $l i$ has been capable [of use in governing] the state; it exists alongside Heaven and Earth. [When] the ruler commands, ministers are reverent, fathers are kind, sons filial... daughters-in-law submissive; this is ritual. The ruler commands and yet does not violate [rules], ministers are reverent and yet not two-faced... daughters-in-law are submissive and tactful: $l i$ is best [for managing] affairs... The former kings received it from Heaven and Earth to rule their people 5 ."

4. Sur ce système du sacrifice en Chine ancienne, voir Levi (2009).

5. Cité dans Pines (2002): 101-102 (Pines utilise la traduction de James Legge). 
Selon cette nouvelle interprétation «humaniste», les déités auxquelles les rites s'adressent se comportent déjà comme une armée de fonctionnaires:

When the state is to prosper, numinous deities arrive to inspect its virtue. When the state is to be ruined, deities arrive as well to examine its wickedness... I heard that when a state is to prosper, [rulers] listen to the people; when it is to perish they listen to the deities. The deities are all-hearing, all-seeing, upright and correct, and they are consistent. Their behavior depends on that of the human beings.

The people are masters of the deities. Therefore, sage kings carried out the people's affairs first, and then attended to the deities... Devote your efforts to the three seasons, improve the five teachings, let [the people] treat appropriately the nine grades of relatives, and then perform sacrifices. Under such conditions people will be peaceful, and the deities will bestow good fortune... Nowadays, however, everyone has a heart of his own, and the spirits and deities lack their master. Though your [sacrifices] are lavish, what good fortune can be achieved in this way ${ }^{6}$ ?

Si les textes que nous venons de citer montrent ce qu'on pourrait qualifier de «rationalisme humaniste», d'autres textes de la même époque permettent de voir que l'intériorisation va de pair avec la rationalisation:

The regulations and patterns of ritual were created based on human affective dispositions, in order to serve as an embankment dam for the people.

In carrying out the... sacrifices to the ghosts and spirits, if it is not ritually proper, then there will be neither sincerity nor gravity. This is why the gentleman is respectful, reverent, frugal, retiring, and yielding in order to clarify the ritual.

If one gives birth to reverence in the state, the [people of] the state will all submit. They will submit only if there is reverence, there will be reverence only if there is yielding, there will be yielding only if there are rites.

For all those who work at persuasion and governing, nothing is as good as sincerity ${ }^{7}$.

Ainsi, le rite ayant été «instrumentalisé » pour inculquer aux uns la sincérité et aux autres la soumission, qu'importe la présence réelle?

Dans tout cela, l'idée la plus intéressante est celle qui veut que les déités soient «consistent»: logiques, cohérentes avec elles-mêmes, en un mot, rationnelles, comme la bureaucratie qui se met en place. On voit se poindre une société composée d'une élite de sujets « sincères» et rationnels et d'un peuple obéissant mais

6. Ibid.: 78, 76-77.

7. Csikszentmihàlyi (2009): t. 1: 524, 527, 531 et 537. Les deux premières citations proviennent du Liji (Mémoire des rites), la troisième du Livre perdu des Zhou (Yi Zhoushu) et la dernière du Lüshi chunqiu. 
écouté, là où, dans l'ancienne société royale, il y avait une élite de «mangeurs de viande» et un «mandat céleste» ${ }^{8}$. Désormais, pourrait-on dire, vox Dei vox populi.

Ainsi, vidé de sa substance sociale royale et converti en instrument pédagogique, le rite sacrificiel allait demeurer au cœur du dispositif de l'empire bureaucratique. Parallèlement à cette transformation des rites anciens sera menée une campagne de dénigrement sans merci contre les officiants de la religion des Zhou, les «chamanes» $(w u)^{9}$, de plus en plus perçus comme les prêtres d'une religion «populaire». Mais l'empire fondé en 221 avant notre ère n'avait pas encore de religion universelle nouvelle et continua donc à avoir recours aux chamanes ${ }^{10}$. C'est lorsque la première expérience impériale aura échoué que le bouddhisme viendra suppléer à ce manque, en introduisant de nouvelles formes de société et de logique «inimaginables» (buke siyi). La société, c'est le sangha ou communauté de moines. Pour bien marquer la rupture que constituait cette nouvelle forme de société, les Chinois donnèrent aux moines le sobriquet de gens ayant «quitté la famille» (chujia), chose proprement inimaginable dans la Chine du culte des ancêtres et de la piété filiale. La logique, aussi implacable que celle des déités évoquées ci-dessus mais ô combien plus universelle (et individuelle), c'était le karma: chaque acte avait sa conséquence qui poursuivait l'individu à travers ses vies successives jusqu'à ce que toute dette fût payée. Mais, plus positivement, là où les dieux anciens agissaient en écho au comportement humain, la divinité bouddhiste la plus populaire, Guanshiyin, répondait au moindre vœu murmuré, même mentalement, par ses dévots. Comme dans le christianisme, la pédagogie rituelle fut soutenue par une riche imagerie des paradis et des enfers et, tout comme Dieu, le Bouddha se trouvait en tout un chacun. Ainsi, à chaque extrémité du continent asiatique, naissait une religion de salut universel qui allait venir au secours d'un empire moribond en déployant une économie du don et de la charité. Le bouddhisme contribua aussi de manière ostentatoire au mouvement masculinisant de l'histoire car, pour être sauvée, une femme devait d'abord revêtir le corps d'un homme - et le moine, évidemment, rompre avec la sexualité et tout ce qui pouvait lui rappeler qu'il était un corps né d'une femme. Même les formes les plus radicalement platoniciennes de la théologie chrétienne sont «matérialistes» en comparaison avec l'idéalisme (weixin) bouddhiste.

Mais nous n'avons voulu, ici, évoquer le bouddhisme que pour relire le paragraphe de Yu Ying-shih cité ci-dessus. On peut notamment comprendre

8. Sur les «mangeurs de viande » et la nouvelle bureaucratie déterritorialisée, voir l'article de Jean Levi cité en note 4 ci-dessus.

9. Lin (2009).

10. Bujard (2009). 
maintenant que les moines bouddhistes étaient pour les gens du Daoxue (T'aohsïeh) ce qu'étaient les chamanes pour l'élite des Royaumes combattants: les représentants de la vieille religion qu'il convenait de vider de sa substance pour en prendre la logique et la «laïciser». À la «compassion» (ci) bouddhique, les nouveaux confucéens substituaient la bonne vieille vertu de ren, de l'«humanité». Mais, si le ren n'était que primus inter pares dans le confucianisme ancien, il était désormais la vertu qui englobait toutes les autres: le résumé intégral et suffisant du comportement idéal du sage. Mais, après presque mille ans de bouddhisme, tout le monde pouvait devenir sage car tout le monde avait la même «nature humaine» (xing) et le même «cœur bon» (liangxin). Il suffisait, pour devenir sage, d'étudier les Quatre livres (sishu), voire, dans les formes les plus bouddhisées du néo-confucianisme (Lu Jiuyuan, Wang Yangming), de s' «éveiller», de se rendre compte que l'on était «sage» depuis toujours. Il n'y avait rien à faire, juste à rester dans ce que l'un des Quatre livres appelait le weifa, le temps de la quiétude (jing) avant que les désirs ne naissent: la sortie de soi qu'est le désir (yifa) doit toujours être surdéterminée par le weifa, exactement comme dans le taoïsme ancien (chang you yu yi jian qi jiao).

Ce nouveau confucianisme, de nationalisme marginal allait devenir orthodoxie nationale. Il allait surtout réinventer la société chinoise en étendant au peuple les mêmes sacrifices aux ancêtres qui, dans l'Antiquité, définissaient l'élite et étaient donc explicitement interdits au peuple: démocratisation. Mais la forme sociale qui allait en naître, le clan (zongzu), était une organisation masculine et les femmes étaient exclues du nouveau culte aux ancêtres. La charité du sangha fut convertie en charité du clan, et servait entre autres à encourager les veuves à rester dans le clan, à ne pas se remarier après la mort de leur mari, même lorsque celle-ci intervenait quand la «veuve» était encore enfant. Ainsi se développa de manière prodigieuse le culte de la femme chaste, faisant écho à cet autre culte de la chasteté virginale, celui de Marie. C'est pendant la même période de l'histoire chinoise que le bandage des pieds des femmes devient universel (ou presque), autrement dit que la femme devient objet esthétique: fétiche.

Cependant, si la fin de l'histoire impériale chinoise est celle d'un patriarcalisme sans concessions, le «pli» propre à la culture chinoise ne disparaît pas pour autant: plus que jamais, la calligraphie était l'art par excellence des lettrés, et le rite demeurait un souci central des néo-confucéens, ainsi qu'on peut le voir par le fait que leur Thomas d'Aquin, Zhu Xi (1123-1200), n'écrivit non pas une somme théologique mais le Jiali, ou Rites pour les familles ${ }^{11}$. On pourrait même aller plus loin et dire que le matérialisme marxiste et désormais capitaliste de la Chine depuis 1949 ainsi que son rapport à l'espace - la «terre

11. Voir Ebrey (1991a et 1991b). 
sacrée » de la «patrie ancestrale» (zuguo) - demeurent profondément «pliés». En tout état de cause, le «communisme confucéen» aura réalisé, au nom du Peuple (et pendant un temps?), la destruction rêvée depuis plus de deux mille ans de la religion populaire.

Le matérialisme universel qui règne aujourd'hui sur la planète peut sembler représenter un renversement de l'histoire idéaliste et patriarcale. Est-ce ainsi ? Met-il vraiment fin au dualisme traditionnel à tendance masculine? Insistons d'abord sur le fait qu'il ne met aucunement fin au dualisme puisque, dans les deux formes de matérialisme que nous venons d'évoquer, l'idéologie joue un rôle central. Même, puisque l'économie de marché a voulu se penser comme «auto-régulante» et le matérialisme marxiste comme sortie utopique de l'histoire - dans la mesure où ils se sont pensés tous deux comme «synthèse universelle et définitive», comme «sortie de la religion»-, ils représentent, au contraire, l'ultime expression de l'idéologie du Père, où la «réalité rugueuse » n'est plus à étreindre puisque les valeurs universelles s'imposent désormais comme une évidence mathématique. L'homme (le genre masculin), s'imaginant toujours plus autonome, «humaniste», a cru pouvoir, enfin, se débarrasser des prêtres et autres médiateurs des religions antiques. Mais, comme par le passé, il n'a réussi qu'à troquer une religion - un système de valeurs - et une série de médiateurs pour une autre.

Pour survivre, maintenant que les dualismes chinois et occidentaux (entre autres) se sont rencontrés, il va bien falloir que les humains acceptent, humblement, dirais-je, qu'il n'y a pas de sortie de la religion possible, que nous ne sommes pas autonomes, que nos actes et nos choix de valeurs, toujours médiatisés par l'histoire et la culture, auront toujours des conséquences «écologiques». Nous aurons toujours à payer nos dettes. 
Dieu-Père/Dao-Mère: dualismes occidentaux et chinois

\section{BibLIOGRAPHIE}

BANON David (1987). La Lecture infinie. Les voies de l'interprétation midrachique. Paris:

Éditions du Seuil.

BARR James (1973). The Bible in the Modern World. Londres: SCM Press.

BILLETER Jean-François (1989). L'Art chinois de l'écriture. Genève: Skira.

Bujard Marianne (2009). Cultes d'État et cultes locaux dans la religion des Han. In John

LAGERwey (dir.), Religion et société en Chine ancienne et médiévale. Paris : Éditions du Cerf.

Cheung Anthea (2010). Le Wuwei chez Guo Xiang : origines, évolution et apogée. Thèse de doctorat, Paris: École Pratique des Hautes Etudes.

Chouraqui André (trad.) (1985). La Bible Chouraqui. Paris: Desclée de Brouwer.

Csirszentminàly Mark (2009). Ethics and Self-cultivation Practice in Early China. In

John Lagerwey et Marc Kalinowski (dir.), Early Chinese Religion, Part One:

Shang through Han. Leyde: Brill.

Derrida Jacques (1967). De la grammatologie. Paris: Les Éditions de Minuit.

Derrida Jacques (1972). La Voix et le phénomène. Paris: Presses Universitaires de France.

Ebrey Patricia (1991a). Confucianism and Family Rituals in Imperial China : A Social History of Writing about Rites. Princeton: Princeton University Press.

Ebrey Patricia (trad.) (1991b). Chu Hsi's Family Rituals: A Twelfth-Century Chinese Manual for the Performance of Cappings, Weddings, Funerals, and Ancestral Rites. Princeton: Princeton University Press.

Foulk Griffith (1999). Song Controversies Concerning the "Separate Transmission" of Chan. In Peter N. Gregory et Daniel A. Getz (dir.), Buddhism in the Song. Hawaii : University of Hawaii.

KaLinowsKi Marc (1985). La transmission du dispositif des neuf palais sous les Six Dynasties. In Michel Strickmann (dir.), Tantric and Taoist Studies in Honour of R.A. Stein, vol. III. Bruxelles: Chiois.

KaLINOWSKI Marc (2009). La divination sous les Zhou orientaux (770-256 avant notre ère).

Textes transmis et découvertes archéologiques récentes. In John LAGERWEY (dir.), Religion et société en Chine ancienne et médiévale. Paris: Éditions du Cerf.

LAGERWEY John (1981). Wu-shang pi-yao: somme taoïste du vie siècle. Paris: École française d'Extrême-Orient.

LAGERwEy John (2004). Deux écrits taoïstes anciens. Cahiers d'Extrême-Asie, no 14 : 139-171.

LeVI Jean (2009). Le rite, la norme et le Tao: philosophie du sacrifice et transcendance du pouvoir en Chine ancienne. In John Lagerwey (dir.), Religion et société en Chine ancienne et médiévale. Paris : Éditions du Cerf.

Levi Jean (trad.) (2010), Les Euvres de Maître Tchouang. Paris : Éditions de l'encyclopédie des nuisances.

LiN Fu-shih (2009). Early Medieval Shamans and Politics. In John Lagerwey et Marc Kalinowski (dir.), Early Chinese Religion, Part One : Shang through Han. Leyde: Brill. 


\section{John Lagerwey}

Madsen Richard (2010). The Upsurge of Religion in China. Journal of Democracy, vol. XXI, no 4 : 58-71.

Mollier Christine (2006). Visions of Evil: Demonology and Orthodoxy in Early Taoism. In Benjamin Penny (dir.), Daoism and History, Essays in Honour of Liu Ts'un-yan. Londres: Routledge.

Mollier Christine (2008). Buddhism and Taoism Face to Face: Scripture, Ritual, and Iconographic Exchange in Medieval China. Honolulu: University of Hawaii Press.

Mollier Christine (2011). Taoïsme médiéval et bouddho-taoïsme : écrits, rituels et images en contexte, document de synthèse pour le dossier scientifique d'Habilitation à diriger des recherches, soutenu le 22 janvier 2011.

PINES Yuri (2002). Foundations of Confucian Thought: Intellectual Life in the Chunqiu Period, 722-453 B.C.E. Honolulu: University of Hawaii Press.

Scholem Gershom (1977). Les Grands Courants de la mystique juive. Paris: Payot.

VANDERMEERSCH Léon (1980). Wangdao ou la Voie royale: recherches sur l'esprit des institutions de la Chine archä̈que. T. 2 : Structures politiques. Les rites. Paris: École française d'Extrême-Orient.

Yu Ying-shih (1992). Foreward. In Hoyt Cleveland Tillman (dir.), Confucian discourse and Chu Hsi's Ascendancy. Honolulu: University of Hawaii Press. 
Dieu-Père/Dao-Mère: dualismes occidentaux et chinois

\section{GLOSSAIRE}

buke siyi 不可思義

Cantongqi 參同契

chujia 出家

$c i$ 慈

Daoxue 道學

$f u$ 符

Huangshu guodu yi 黃書過度儀

Huangting jing 黃庭經

Hundun 混沌

$j i$ 跡

Jiali 家禮

jing 靜

Laozi zhong jing 老子中經

li 理

$l i$ 禮

liangxin 良心

Lu Jiuyuan 陸九淵

neidan 內丹

neiguan 內觀

qiangming 強名

ren 仁

Shenzhou jing 神呪經

Sishu 四書

$\tan$ 壇

ti Dao 體道

weifa 未發

$w u$ 巫

Wushang biyao 無上秘要

Wuzhen pian 悟真篇

xiaoyao you 逍遙遊

xing 性

yifa 已發

Yijing 易經

yuanqi 元氣

yu Dao hezhen 與道合真

Zhuangzi 莊子

Zhu Xi 朱喜

zongzu 宗族

zuguo 祖國

Zuozhuan 左傳 
